\title{
Metodología para evaluar el nivel ético en las organizaciones
}

\author{
Methodology for assessing ethical level in organizations \\ Juan Antonio Plasencia Soler ${ }^{1 *} \quad$ Fernando Marrero Delgado ${ }^{2} \quad$ Miriam Nicado García $^{1}$ \\ Recibido 21 de septiembre de 2015, aceptado 4 de abril de 2016 \\ Received: September 21, $2015 \quad$ Accepted: April 4, 2016
}

\begin{abstract}
RESUMEN
La ética organizacional en la actualidad se erige como un referente en la gestión de las empresas que desean obtener resultados en un mundo cada vez más competitivo y globalizado, a pesar de que muchas organizaciones utilicen estos métodos de gestión para encubrir un objetivo superior, que no es otro que la maximización de los ingresos.

La presente investigación propone por medio de los métodos: Análisis de Modos de Fallas, Efectos y Criticidad (AMFEC) y el Proceso de Jerarquía Analítica (AHP) y basada en los sistemas, normas e indicadores propuestos por diversas instituciones internacionales, una metodología para evaluar el nivel ético de una organización.

La metodología posibilita la construcción de una jerarquía compuesta por dimensiones, factores críticos e indicadores aplicando el método AMFEC, el que permite evaluar cada uno de los elementos según su consecuencia, frecuencia de ocurrencia y detección. El método se complementa al determinar los pesos de los elementos mediante el AHP utilizando una escala fundamental de valores.

La metodología propuesta determina el nivel ético en el impacto de sus dimensiones, así como evalúa mediante una escala el comportamiento ético de una organización, permitiendo valorar los efectos generados por las organizaciones en la sociedad, el medio ambiente y la economía.
\end{abstract}

Palabras clave: Ética organizacional, análisis de criticidad, proceso de jerarquía analítica, nivel ético organizacional.

\begin{abstract}
Nowadays Business ethics emerge as a referent in management into the organizations that want to obtain outcome in a competitive and globalized world, despite there are many companies that used these management methods for cover a top objective that is profit maximization.

This research proposes through the methods: Failure Modes, Effects and Criticality Analysis (FMECA) and the Analytic Hierarchy Process (AHP), and systems, standards and indicators proposed by various international institutions a methodology for assessing the ethical level in an organization.

The methodology enables the construction of a hierarchy composed of dimensions, critical factors and indicators using the FMECA method, which allows to evaluate each of the elements according to their severity, occurrence and detection. The method is complemented when determining the weights of the elements through AHP using a basic scale of values.

The proposed methodology determines the ethical level through the impact of its dimensions, and a fundamental scale evaluates the ethical behavior allowing to measure the effects generated by organizations in society, the environment and the economy.
\end{abstract}

Keywords: Business ethics, criticality analysis, analytic hierarchy process, ethical level organizational.

1 Departamento de Ciencias Empresariales. Universidad de las Ciencias Informáticas. Carretera a San Antonio de los Baños, Km. 2 1⁄2. Torrens, municipio de La Lisa. La Habana, Cuba. E-mail: juanps@uci.cu; nicado@uci.cu

2 Facultad de Ingeniería Industrial y Turismo. Universidad Central "Martha Abreu" de las Villas. Carretera a Camajuaní Km. 5 y 1/2. Santa Clara. Villa Clara, Cuba. E-mail: FMarrero@uclv.edu.cu

* Autor de correspondencia 


\section{INTRODUCCIÓN}

La empresa es reflejo del entorno en el que se desenvuelve, pero también juega un papel importante en la trasformación de esa realidad que le rodea, de establecer modos de actuación correctos, de generar beneficios, de satisfacer, en fin, necesidades económicas, sociales y medioambientales.

En los últimos años ha existido un recio debate sobre el papel de las empresas ante los problemas sociales y su razón de ser más allá de la maximización de los ingresos, involucrándose en lo que algunos llaman externalidades económicas.

Esta es una época caracterizada por una serie de problemáticas a nivel internacional, donde la forma de gestionar los negocios tienen una gran responsabilidad, así por ejemplo, la distribución de la riqueza de la población mundial es cada vez menos equitativa [1]. El desempleo a nivel mundial se ha mantenido en $6 \%$ de la población activa, pero en países como España asciende a $22 \%$, y a un astronómico $49,6 \%$ de los menores de 25 años [2-3].

El número de mamíferos, aves, reptiles, anfibios y peces en todo el planeta es, en promedio, la mitad de lo que era hace 40 años, y por si fuera poco la huella ecológica aumenta desproporcionadamente en comparación con la biocapacidad del planeta, provocando que sean necesarios 1,5 planetas para satisfacer la demanda de la población actual [4].

Estas problemáticas hacen que cada día con mayor fuerza las empresas se preocupen más por los efectos que generan en la economía, la sociedad y el medioambiente. Estos efectos tienen una estrecha relación con la ética organizacional y específicamente con una de sus vertientes: la responsabilidad social corporativa (RSC).

El objetivo de esta investigación es proponer una metodología para evaluar el nivel ético de una organización combinando el Análisis de Modos de Fallas, Efectos y Criticidad (AMFEC) y el Proceso de Jerarquía Analítica (AHP).

Para cumplir este objetivo, los autores han divido el estudio en dos partes: la primera, un marco teórico sobre los niveles de la ética empresarial; las normas, indicadores y sistemas utilizados para evaluar ética, responsabilidad social y sostenibilidad; y los métodos AMFEC y el AHP, y, una segunda parte donde se propone una metodología para evaluar el nivel ético de una organización.

\section{MARCO TEÓRICO}

\section{Niveles de la ética empresarial}

La ética empresarial puede ser examinada desde cuatro niveles básicos. Estos niveles para un mejor entendimiento de las complejas relaciones que pueden presentar los problemas éticos se clasifican en nivel individual o personal, nivel organizacional o de compañía, nivel industrial o nacional y, por último nivel internacional o de sociedad [5-7]. Se ofrece una breve descripción de estos niveles en la Tabla 1.

La ética empresarial tiene además una dimensión interna y otra externa, por lo que medir el comportamiento ético de una organización debe estar en correspondencia, en primer lugar, con el actuar de sus procesos internos, y el comportamiento de sus trabajadores y, en segundo lugar, con el impacto de sus resultados en la sociedad, el medio ambiente y en la economía [8]:

- En el ámbito interno pone especial atención en la demanda ética por parte de trabajadores y directivos a todos los niveles, exigiendo la puesta en práctica de una serie de valores compartidos, normas, regulaciones y evitando las malas prácticas en las operaciones diarias.

- En la dimensión externa la empresa enfrenta continuamente conflictos de interés que tienen que ver con la calidad de sus servicios, las relaciones con sus suministradores, clientes, accionistas, y otras partes interesadas en el negocio, exige también una actitud responsable con la comunidad y el medio ambiente que le rodea.

Por otra parte, la ética de una organización, se ocupa de estudiar los presupuestos que subyacen a la credibilidad o legitimidad social de esta, de analizar la extensión de su responsabilidad social y ecológica [9].

Los autores de esta investigación defienden este concepto donde la responsabilidad social corporativa es parte de la ética empresarial. Muy ligado a este concepto de responsabilidad social empresarial está 
Tabla 1. Niveles de la ética empresarial.

\begin{tabular}{|l|l|}
\hline $\begin{array}{c}\text { Niveles de la ética } \\
\text { empresarial }\end{array}$ & \multicolumn{1}{c|}{ Descripción } \\
\hline Nivel individual & $\begin{array}{l}\text { Incluye situaciones de conflicto que el individuo encara en la vida personal, puede ocurrir } \\
\text { como resultado de presiones de trabajo o motivaciones o circunstancias no relacionadas } \\
\text { con el trabajo. }\end{array}$ \\
\hline Nivel organizacional & $\begin{array}{l}\text { Se refiere al actuar ético de la organización, en sus roles las personas confrontan cuestiones } \\
\text { éticas, en las que el reconocimiento de circunstancias de malas prácticas organizacionales } \\
\text { son usualmente el resultado de un conjunto de decisiones inapropiadas. Estas decisiones } \\
\text { traen consecuencias para la reputación de la empresa, el éxito o no en la comunidad, y } \\
\text { en el clima ético y cultural que prevalece en la organización. En este nivel, los miembros } \\
\text { de una organización deberán examinar las políticas, procedimientos y códigos de ética } \\
\text { existentes antes de tomar decisiones o realizar acciones. }\end{array}$ \\
\hline Nivel industrial & $\begin{array}{l}\text { Se refiere al conjunto de prácticas éticas: normas, valores, código de ética, que guían el } \\
\text { comportamiento de los trabajadores de determinada industria, así como los resultados y } \\
\text { efectos finales del comportamiento corporativo que se considera apropiado o inapropiado. }\end{array}$ \\
\hline Nivel internacional & $\begin{array}{l}\text { Este incluye los niveles industrial, organizacional e individual. Incluye leyes, normas, } \\
\text { costumbres y tradiciones que rigen el comportamiento legal y moral de una sociedad. }\end{array}$ \\
\hline
\end{tabular}

el enfoque de resultado triple, donde para lograr un desarrollo sostenible, los negocios deberán cumplir no solo objetivos económicos, sino también sociales y medioambientales [10-16].

A continuación, los autores hacen referencia a algunas normas, sistemas e indicadores propuestos para evaluar la ética en las organizaciones.

\section{Propuestas para evaluar la ética empresarial}

Existen no pocos esfuerzos desde hace varios años dirigidos a evaluar la ética empresarial, por lo que se han desarrollado normas, sistemas e indicadores diseñados por prestigiosas instituciones internacionales [17-26].

Estos proponen un grupo de dimensiones, requisitos, temas, subtemas e indicadores para que las organizaciones puedan evaluar el nivel de compromiso social, económico y medioambiental.

En la Tabla 2 se muestra un resumen de algunas normas, sistemas e indicadores para evaluar la ética en las organizaciones.

Teniendo en cuenta que para evaluar el nivel ético de una institución es necesario construir un índice, compuesto por indicadores asociados a factores críticos y estos a su vez a dimensiones, todas estas variables en forma de jerarquía. Los autores proponen combinar el método de Análisis de Modo de Fallas,
Efectos y Criticidad (AMFEC) con el Proceso de Jerarquía Analítica (AHP).

\section{Método AMFEC}

El AMFEC es un método utilizado para establecer jerarquías entre sistemas, instalaciones, equipos y partes de equipos [27-29].

Este método es muy utilizado en la priorización de riesgos; partiendo de un análisis funcional, es posible luego identificar los modos de fallas, efectos y consecuencias para jerarquizar los riesgos $[27,30]$.

Concibiendo la organización como un sistema, se propone utilizar este método para construir la jerarquía para determinar el nivel ético organizacional.

La jerarquía se construye según el índice de prioridad del riesgo (IPR) o índice de criticidad (IC). Las dos formas más comunes utilizadas en el cálculo de este índice se muestran las ecuaciones siguientes (1) y (2) [27,31-32]:

$$
\begin{gathered}
I C \text { о IPR }=C * P \\
I C \text { о IPR }=C * P * D
\end{gathered}
$$

Donde:

$C$ : Consecuencia o gravedad.

$P$ : Frecuencia o probabilidad de ocurrencia.

$D$ : Detección. 
Tabla 2. Resumen de propuestas para evaluar ética organizacional.

\begin{tabular}{|l|c|l|l|}
\hline \multicolumn{1}{|c|}{$\begin{array}{c}\text { Normas/ Indicadores/ } \\
\text { Índices }\end{array}$} & Año & \multicolumn{1}{|c|}{ Temática } & \multicolumn{1}{|c|}{ Institución } \\
\hline $\begin{array}{l}\text { Dow Jones Sustainability } \\
\text { World Index }\end{array}$ & 1999 & $\begin{array}{l}\text { Índice para evaluar la sostenibilidad de las } \\
\text { compañías líderes mundiales }\end{array}$ & REBECOSAM \\
\hline FTSE4Good Index Series & 2001 & $\begin{array}{l}\text { Índice diseñado para medir el rendimiento } \\
\text { sostenible de las compañ́as }\end{array}$ & London Stock Exchange Group \\
\hline Ethibel Sustainability Index & 2004 & $\begin{array}{l}\text { Índice que permite evaluar la sostenibilidad } \\
\text { de las empresas }\end{array}$ & Forum Ethibel \\
\hline Ethisphere & 2007 & $\begin{array}{l}\text { Sistema que permite mediante un coeficiente } \\
\text { ético evaluar el comportamiento de las } \\
\text { empresas }\end{array}$ & Ethisphere Institute \\
\hline SGE 21 & 2008 & $\begin{array}{l}\text { Norma para la evaluación de la gestión ética y } \\
\text { socialmente responsable en las organizaciones }\end{array}$ & $\begin{array}{l}\text { Forética. Foro para la Evalua- } \\
\text { ción de la Gestión Ética }\end{array}$ \\
\hline AA1000 AS & 2008 & Norma de aseguramiento de sostenibilidad & AccountAbility \\
\hline SA8000 & 2008 & Norma internacional de responsabilidad social & $\begin{array}{l}\text { Social Accountability Interna- } \\
\text { tional (SAI) }\end{array}$ \\
\hline ISO 26000 & 2010 & $\begin{array}{l}\text { Noma internacional sobre responsabilidad } \\
\text { social }\end{array}$ & $\begin{array}{l}\text { International Organization for } \\
\text { Standardization (ISO) }\end{array}$ \\
\hline GRI G4 Guidelines & 2013 & $\begin{array}{l}\text { Guía para la elaboración de reportes de } \\
\text { sostenibilidad }\end{array}$ & $\begin{array}{l}\text { Global Reporting Initiative } \\
\text { (GRI) }\end{array}$ \\
\hline Ethos & 2014 & $\begin{array}{l}\text { Indicadores para evaluar negocios sustentables } \\
\text { y responsables }\end{array}$ & Instituto Ethos \\
\hline
\end{tabular}

Tanto la frecuencia de ocurrencia, la probabilidad de detección, así como las consecuencias del evento pueden ser determinadas por métodos objetivos y subjetivos.

\section{Proceso de Jerarquía Analítica}

El AHP es un método desarrollado por Thomas L. Saaty a principios de la década de los años 80 del siglo pasado. Este método denominado: proceso de jerarquía analítica, incluye los pasos siguientes [33-35]:

1. Definir el problema y determinar la clase de conocimiento buscado.

2. Estructurar la jerarquía de decisión desde arriba con la meta de la decisión, luego los objetivos desde una perspectiva amplia por medio de niveles intermedios (criterios sobre los que dependen los elementos subsiguientes) hacia los niveles más bajos (lo usualmente es un conjunto de alternativas).

3. Construir un conjunto de matrices de comparación de pares de juicio. Cada elemento de un nivel superior es usado para comparar los elementos en el nivel inmediatamente inferior con respecto a estos.
4. Usar las prioridades obtenidas desde las comparaciones para ponderar las prioridades en el nivel inmediatamente inferior. Esto se hace para cada elemento. Luego para cada elemento del nivel inferior se añaden estos valores de pesos y se obtiene la prioridad global. Continuar el proceso de ponderación hasta obtener las prioridades finales de las alternativas en el nivel más bajo.

Para hacer las comparaciones es necesario una escala de números que indica cuántas veces es más importante o dominante un elemento sobre otro con respecto al criterio o propiedad con respecto al que ellos son comparados, tal y como se muestra en la Tabla 3.

\section{RESULTADOS}

Para evaluar el comportamiento ético de una organización los autores proponen una metodología que tiene como base las normas, sistemas e indicadores propuesto por prestigiosas organizaciones y enunciados en el epígrafe anterior, además de los métodos: Análisis de Modos de Fallas, Efectos y Criticidad (AMFEC) y el Proceso de Jerarquía Analítica (AHP). 
Tabla 3. Escala de ponderación para la comparación de los criterios [33-34].

\begin{tabular}{|c|c|c|}
\hline $\begin{array}{c}\text { Intensidad de } \\
\text { importancia }\end{array}$ & Definición & Explicación \\
\hline 9 & Extremadamente más preferido & $\begin{array}{l}\text { La evidencia que favorece una actividad sobre } \\
\text { otra es del orden mayor posible de afirmación. }\end{array}$ \\
\hline 8 & De muy poderosamente más a extremadamente más & \multirow{3}{*}{$\begin{array}{l}\text { Una actividad es favorecida muy poderosamente } \\
\text { sobre otra. Su dominancia es demostrada en } \\
\text { la práctica }\end{array}$} \\
\hline 7 & Muy poderosamente más preferido & \\
\hline 6 & De poderosamente más a muy poderosamente más & \\
\hline 5 & Poderosamente más preferido & \multirow{2}{*}{$\begin{array}{l}\text { La experiencia y el juicio están poderosamente } \\
\text { a favor de una actividad sobre otra }\end{array}$} \\
\hline 4 & De moderadamente más a poderosamente más & \\
\hline 3 & Moderadamente más preferido & \multirow{2}{*}{$\begin{array}{l}\text { La experiencia y el juicio favorecen ligeramente } \\
\text { una actividad sobre otra }\end{array}$} \\
\hline 2 & De igual a moderadamente más & \\
\hline 1 & Igualmente preferido & $\begin{array}{l}\text { Las dos actividades contribuyen igualmente } \\
\text { al objetivo }\end{array}$ \\
\hline $\begin{array}{l}\text { Valores } \\
\text { recíprocos }\end{array}$ & $\begin{array}{l}\text { Si la actividad i tiene un valor cuando se comparó } \\
\text { con la actividad j, luego j tiene un valor reciproco } \\
\text { cuando es comparada con } \mathrm{i}\end{array}$ & Una suposición razonable \\
\hline $1.1-1.9$ & Si las actividades son muy cercanas & $\begin{array}{l}\text { Puede ser difícil asignar el mejor valor pero } \\
\text { cuando se compara con otras actividades el } \\
\text { tamaño de los números pequeños puede no } \\
\text { ser evidente, todavía ellas pueden indicar la } \\
\text { importancia relativa de las actividades }\end{array}$ \\
\hline
\end{tabular}

La Figura 1 muestra la secuencia de pasos, propuesta por los autores de esta investigación, para determinar el comportamiento ético de una organización.

A continuación, se describen cada uno de los pasos propuestos.

\section{Paso 1. Conformar la jerarquía}

En este paso se realiza un análisis de criticidad, basada en la metodología de Análisis de Modos de Falla, Efectos y Criticidad (AMFEC).

Los autores proponen conformar una jerarquía teniendo como meta el nivel ético $(\mathrm{Ne})$, al que están asociado dimensiones (Dim), a las que se asocian factores críticos $(C)$ y a estos indicadores $(I)$ tal y como se muestra la ecuación (3):

$$
N e=N e(\operatorname{Dim}(C(I)))
$$

El procedimiento a seguir para la conformación de la jerarquía es el siguiente:

1. Identificación de las dimensiones y factores críticos que afectan la ética.

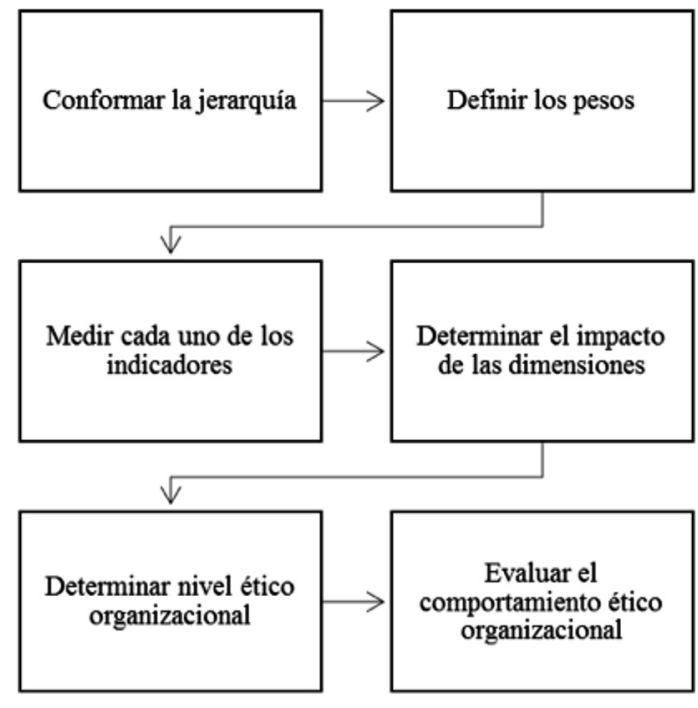

Figura 1. Pasos de la metodología para evaluar el nivel ético organizacional.

2. Determinación de los indicadores asociados a cada factor crítico.

3. Evaluación de las consecuencias, ocurrencias y detección.

4. Cálculo del índice de criticidad. 
Es importante en este paso la selección de los expertos que tienen la responsabilidad de identificar y evaluar las dimensiones, los factores críticos e indicadores que afectan la ética en la organización.

La jerarquía resulta del producto de la frecuencia de ocurrencia, las consecuencias y la detección de cada una de las variables definidas, tal y como muestra la ecuación (2). Existen una gran variedad de escalas que permiten evaluar el grado de consecuencia, ocurrencia y detección de cada elemento de la jerarquía [30, 36-39].

Según los resultados del (IC) estas categorías son ordenadas de mayor a menor, para determinar cuáles son las dimensiones, factores críticos e indicadores más determinantes en el nivel ético de una organización.

\section{Paso 2. Definir los pesos}

Luego de definidas las dimensiones, factores críticos e indicadores, para calcular el nivel ético de la empresa, se deben ponderar los mismos, para ello se utiliza el Proceso de Jerarquía Analítica de comparación por pares, donde por medio de expertos se evalúa la dominancia de una variable sobre otra.

Se recomienda en esta fase utilizar el software Expert Choice para una mejor agilidad y fiabilidad en el cálculo de los pesos.

\section{Paso 3. Medir cada uno de los indicadores}

Asociado a las nuevas misiones en la empresa, no solo como generadora de ganancias, sino también como generadora de bienestar social y ambiental, surgen, un grupo de indicadores relacionados con el medio ambiente y el impacto de la gestión empresarial sobre los recursos naturales, y el uso de estos recursos sin comprometer los recursos de las próximas generaciones [40].

Asimismo, existen indicadores para evaluar el impacto de la gestión empresarial en los trabajadores y la comunidad que le rodea [41-42]. La empresa se identifica en la actualidad como agente de cambios, no solo económicos, sino social y medioambiental.

Una vez calculado los pesos relativos a las dimensiones, los factores críticos y los indicadores, se procede a medir cada uno de los indicadores que componen el comportamiento ético de la organización. Los indicadores pueden tener diversas clasificaciones, de acuerdo al tipo de empresa o de negocio que se evalué, además del tipo de información que se posea, estos pueden ser cualitativos o cuantitativos.

Es necesario en este paso conformar un catálogo de indicadores, que entre otros aspectos debe contar con: nombre del indicador, notación, conceptualización, forma de cálculo, quien responde por su medición, valores límites, etc.

\section{Paso 4. Determinar el impacto de las dimensiones}

En este paso se hace necesario normalizar los criterios de medidas de cada uno de los indicadores calculados en el paso 3. Para normalizar cada uno de los criterios de medidas se recomienda utilizar las ecuaciones (4) y (5), según sea el objetivo del decisor.

Para criterios de medidas que se relacionen con indicadores en los que se busca maximizar el resultado:

$$
E I=\frac{V R}{V I}
$$

Para criterios de medidas que se relacionen con indicadores en los que se busca minimizar el resultado:

$$
E I=\frac{V I}{V R}
$$

Donde:

EI: Evaluación del indicador.

$V I$ : Valor ideal del indicador.

$V R$ : Valor real del indicador.

Luego se calcula el impacto de las dimensiones, utilizando la ecuación (6):

$$
\operatorname{Dim}_{k}=\sum_{i=1}^{n} C_{i k} * W_{i k} \text { para } i=1 \ldots n
$$

Donde:

$\operatorname{Dim}_{k}$ : Impacto en las dimensiones.

$C_{i k}$ : Factores críticos asociados a cada una de las dimensiones.

$W_{i k}$ : Pesos asociados a los factores críticos. 
Para determinar $C_{i k}$ se utiliza la ecuación (7):

$$
C_{i k}=\sum_{j=1}^{n} I_{j i k} * W_{j i k} \text { para } j=1 \ldots n
$$

Donde:

$I_{j i k}$ : Indicadores asociados a los factores críticos. $W_{j i k}$ : Pesos asociados a los indicadores.

Paso 5. Determinar el nivel ético organizacional Luego se procede a determinar el nivel ético de la organización, teniendo en cuenta los impactos de las dimensiones, como muestra la ecuación (8):

$$
N e=\sum_{k=1}^{n} \operatorname{Dim}_{k} * W_{k} \text { para } k=1 \ldots n
$$

Donde:

$\mathrm{Ne}$ : Nivel de ética organizacional.

$W_{k}$ : Pesos asociados a cada una de las dimensiones.

\section{Paso 6. Evaluar el comportamiento ético organizacional}

Los valores de las variables: $\operatorname{Dim}, C, I$ y los pesos se expresan luego de normalizarse con valores entre 0 y 1 entonces el nivel ético $(\mathrm{Ne})$ es evaluado entre 0 y 1 en dependencia del comportamiento ético de la empresa ante todos los interesados, clientes, trabajadores, proveedores, competidores, accionistas, y el efecto generado en la economía, la sociedad y el medio ambiente. Mientras más ético sea el comportamiento de la empresa más cerca del valor 1 estará el valor $\mathrm{Ne}$.

Teniendo en cuenta investigaciones precedentes, que evalúan indicadores de sostenibilidad, responsabilidad social y ética empresarial [19, 43-48] los autores proponen una escala para evaluar el nivel ético de una organización tal y como muestra la Tabla 4.

Tabla 4. Escala de evaluación del nivel ético organizacional.

\begin{tabular}{|l|l|}
\hline \multicolumn{1}{|c|}{ Nivel ético } & \multicolumn{1}{|c|}{ Evaluación } \\
\hline $0,90 \leq \mathrm{Ne} \leq 1$ & Muy alto \\
\hline $0,75 \leq \mathrm{Ne} \leq 0,89$ & Alto \\
\hline $0,50 \leq \mathrm{Ne} \leq 0,74$ & Medio \\
\hline $0,35 \leq \mathrm{Ne} \leq 0,49$ & Bajo \\
\hline $0,00 \leq \mathrm{Ne} \leq 0,34$ & Muy bajo \\
\hline
\end{tabular}

Además, en la definición de la escala se tienen en cuenta los siguientes criterios: asignarle una etiqueta al valor numérico permite identificar el extremo positivo y el extremo negativo; cuanto mayor sea el número de categorías, mayor será la precisión al evaluar el nivel ético, se sugieren escalas de 5 , 7 o 9 categorías; y el uso de un número impar de categorías permite obtener una evaluación neutra o media.

\section{CONCLUSIONES}

El análisis de criticidad puede ser aplicado en la determinación de prioridades de una serie variables, no solo cuando se trata de riesgos, sino también cuando es necesario construir jerarquías en sistemas, como es el caso de una organización.

En esta investigación los autores proponen determinar mediante AMFEC una jerarquía compuesta por dimensiones, factores críticos e indicadores, por medio de una valoración de expertos y calculando luego su Índice de Criticidad para definir los elementos que permitan luego evaluar el nivel ético de una organización.

Se robustece el análisis de criticidad combinándolo con el Proceso de la Jerarquía Analítica (AHP), lo que permite que una vez conformada la jerarquía, se ponderen cada uno de los elementos que la conforman, determinando mediante comparaciones pareadas, qué dimensión, factor crítico e indicador tiene mayor influencia sobre el nivel ético.

La integración de ambos métodos permite modelar un problema complejo de la ciencia actual debido a la composición de las variables cualitativas y cuantitativas y los juicios subjetivos emitidos por expertos, todos componentes involucrados en el proceso de la evaluación del nivel ético.

La metodología propuesta constituye una herramienta novedosa y actual para determinar y evaluar el nivel de ética de una organización. La misma se compone de seis pasos integrados que permiten de manera sencilla su aplicación en cualquier tipo de organización.

La metodología actualmente se aplica en varias organizaciones con muy buenos resultados los que se publicarán como parte de una nueva investigación. 


\section{REFERENCIAS}

[1] Credit-Suisse. "Global Wealth Report", pp. 24. Fecha de actualización: octubre de 2014. Fecha de consulta: 27 de julio de 2015. URL: http://economics.uwo.ca/people/ davies_docs/credit-suisse-global-wealthreport-2014.pdf

[2] Banco Mundial. "Desempleo, total (\% de la población activa total) (estimación modelado OIT)". Fecha de consulta: 27 de julio de 2015. URL: http://datos. bancomundial.org/indicador/SL.UEM.TOTL. ZS/countries?display=graph

[3] Datosmacro. "Desempleo de España". Fecha de consulta: 11 de junio de 2015. URL: http:// www.datosmacro.com/paro/espana

[4] WWF. "Living Planet Report. World Wide Fund Nature", pp. 1-180. Fecha de actualización: 2014. Fecha de consulta: 20 de julio de 2015. URL: https://www.wwf. or.jp/activities/lib/lpr/WWF_LPR_2014.pdf

[5] G. McDonald. "Business Ethics: A Contemporary Approach". Cambrige University Press. Melbourne. Australia. First Edition, pp. 451. 2015. ISBN: 978-1-107-67405-9.

[6] R.R. Sims. "Ethics and Corporate Social Responsibility: Why Giants Fall”. Praeger Publishers. Westport. USA First Edition, pp. 328. 2003. ISBN: 0-275-98039-1.

[7] J.A. Weiss. "Stakeholder and Issues Management Approach". Cengage learning. Mason. USA. 5th Edition, pp. 552. 2015. ISBN: 0-324-58973-5.

[8] E. Ruiz-Otero, M.L. Gago-García, C. GarcíaLeal, S. López-Barra. "Recursos humanos y responsabilidad social corporativa". McGraw Hill Editorial. First Edition. Madrid. España, pp. 288. 2015. ISBN: 8448175646.

[9] D. García-Marzá, "Ética empresarial: del Diálogo a la Confianza". Trotta S.A. Madrid. España. Primera edición, pp. 284. 2004. ISBN: 84-8164-694-6.

[10] A.L. Andrady. "Plastics and Environmental Sustainability: Fact and Fiction". John Wiley \& Sons. New Jersey. USA. First Edition, pp. 352. 2015. ISBN: 1118312600.

[11] M.A. González-Pérez, L. Leonard. "The UN Global Compact: fair Competition, Environmental, and Labour Justice in
International Markets". Emerald Group Publishing Limited. Bingley. UK. First Edition, pp. 250. 2015. ISBN: 1784412953.

[12] O. Laasch and R. Conaway. "Principles of Responsible Management: Global Sustainability, Responsibility". Cengage Learning. Stanford. USA. First Edition, pp. 550. 2015. ISBN: 1-285-08026-0.

[13] R. Mullerat. "International Corporate Social Responsibility: The Role of Corporations". Kluwer Law International. Alphen aan den Rijn. The Netherlands, pp. 536. 2010. ISBN: 9041125906.

[14] H. Robinson, B. Symonds, B. Gilbertson and B. Ilozor. "Design Economics for the Built Environment: Impact of Sustainability on Project Evaluation". John Wiley \& Sons, Ltd. West Sussex. UK. First Edition, pp. 432. 2015. ISBN: 9780470659090.

[15] H. Washington. "Demystifying Sustainability: Towards Real Solutions". Routledge. New York. USA. First Edition, pp. 248. 2015. ISBN: 978-1-138-81268-0.

[16] B. Willard. "The New Sustainability Advantage: Seven Business Case Benefits of a Triple Bottom Line". New Society Publishers. Gabriola Island. Canadá. 10th Edition, pp. 224. 2012. ISBN: 978-0-86571-712-1.

[17] AccountAbility. "AA1000 Accountability Principles Standard", pp. 1-21. Fecha de actualización: 2008. Fecha de consulta: 27 de julio de 2015. URL: http://www.accountability. org/images/content/3/5/350.pdf

[18] Ethisphere. "Scoring \& Methodology". Fecha de consulta: 11 de julio de 2015. URL: http://ethisphere.com/worlds-most-ethical/ scoring-methodology/

[19] Ethos. "Indicadores Ethos para Negócios Sustentáveis e Responsáveis", pp. 1-106. Fecha de actualización: 2013. Fecha de consulta: 15 de julio de 2015. URL: http://www3. ethos.org.br/wp-content/uploads/2013/08/ Indicadores-Ethos-20131.pdf

[20] Forum Ethibel. "Ethibel Sustainability Indices (ESI) Rulebook", pp. 1-13. Fecha de actualización: 2012. Fecha de consulta: 20 de julio de 2015. URL: http://forumethibel. org/content_files/doc\%2020092011/ESI/ ESIRulebookSeptember2012.pdf

[21] FTSE4Good. "Index Inclusion Rules for the FTSE4Good Index Series”, pp. 1-8. Fecha de 
actualización: 2015. Fecha de consulta: 20 de julio de 2015. URL: http://www.ftse.com/ products/downloads/F4G-Index-InclusionRules.pdf

[22] Global Reporting Initiative (GRI). "G4 Sustainability Reporting Guidelines", pp. 1-94. Fecha de actualización: 2013. Fecha de consulta: 27 de julio de 2015. URL: https://www.globalreporting.org/ resourcelibrary/GRIG4-Part1-ReportingPrinciples-and-Standard-Disclosures.pdf

[23] International Organization for Standardization (ISO). "ISO 26000 Guidance on social responsibility", pp. 1-118. Fecha de actualización: 2010. Fecha de consulta: 15 de junio de 2015. URL: http://www.cnis.gov.cn/ wZgg/201405/P020140512224950899020.pdf

[24] RobecoSAM AG. "CSA Guide - Robe coSAM's Corporate Sustainability Assessment Methodology", pp. 24. Fecha de actualización: 2015. Fecha de consulta: 20 de julio de 2015. URL: http://www.sustainability-indices.com/ images/corporate-sustainability-assessmentmethodology-guidebook.pdf

[25] Social Accountability International (SAI). "Social Accountability 8000", pp. 1-16. Fecha de actualización: 2014. Fecha de consulta: 17 de julio de 2015. URL: http://www. dnvba.com/it/DNV\%20\%20Downloads/ SA8000\%20Standard\%202014.pdf

[26] Forética. "SGE-21 Sistema de Gestión Ética y Socialmente Responsable", pp. 1-28. Fecha de actualización: 2008. Fecha de consulta: 28 de julio de 2015. URL: http://sge21. foretica.org/wp-content/uploads/2011/11/ SGE-21_2008_ES-DEF.pdf

[27] A.M. C-S, M.L. Brito-Ballina y E. Fraga-Guerra. "Análisis de criticidad personalizados". Ingeniería Mecánica. Vol. 12 $\mathrm{N}^{\mathrm{o}}$ 3, pp. 1-12, septiembre-diciembre de 2009. ISSN: 1815-5944.

[28] D. Singh and A. Suhane. "Study of Centrifugal Pump Using Failure Mode Effect and Critical Analysis Based on Fuzzy Cost Estimation: A Case Study". International Journal of Scientific Research (IJSR). Vol. $4 \mathrm{~N}^{\mathrm{o}} 7$, pp. 19-22. Julio de 2015. ISSN: 2319-7064.

[29] L. Y-F, H. Hong-Zhong, Z. X. Hanliang and Yu L. Ning-Cong. "Fuzzy Sets Method of Reliability Prediction and Its Application to a Turbocharger of Diesel Engines". Advances in Mechanical Engineering. Vol. 5. 28 de octubre de 2013. DOI: 10.1155/2013/216192.

[30] J.R. Aguilar-Otero, D. Magaña-Jiménez y R. Torres-Arcique. "Análisis de modos de falla, efectos y criticidad (AMFEC) para la planeación del mantenimiento empleando criterios de riesgo y confiabilidad". Revista Tecnología Ciencia y Educación. Vol. 25 $\mathrm{N}^{\circ}$ 1, pp. 15-26. 2010.

[31] A. Díaz-Concepción, F. Pérez-Rodríguez, A. del Castillo-Serpa y M.L. Brito-Vallina. "Propuesta de un modelo para el análisis de criticidad en plantas de productos biológicos". Ingeniería Mecánica. Vol. 15 No 1, pp. 34-43, enero-abril de 2012. ISSN: 1815-5944.

[32] Z. Shanfeng, L. Mengwei, Z. Haiyan and Z. Ruili. "Aircraft Fuel System Fuzzy FMEA and FMECA Analysis". International Conference on Information Sciences, Machinery, Materials and Energy (ICSMME 2015). Chongqing, China. 11-13 de abril de 2015.

[33] T.L. Saaty. "Decision making with the analytic hierarchy process". International Journal of Services Sciences. Vol. 1. No 1, pp. 83-98. 2008. DOI: 10.1504/ijssci.2008.017590.

[34] Jorge Pérez Rave, Mónica Trujillo and Gloria Castro. "Modelación multicriterio del nivel de prevención de contaminación por mercurio en entidades odontológicas". Ingeniare Revista chilena de ingeniería. Vol. $23 \mathrm{~N}^{\circ}$ 1, pp. 128-144. 2015. DOI: 10.4067/S0718-33052015000100015.

[35] R. Costa, J. Siluk, A. Neuenfeldt Júnior, M. Soliman e E. Nara. "A gestão da competitividade industrial por meio da aplicação dos métodos UP e multicritério no setor frigorífico de bovinos". Ingeniare Revista chilena de ingeniería. Vol. 23 $\mathrm{N}^{\circ} 3$, pp. 383-394. 2015. DOI: $10.4067 /$ S0718-33052015000300007.

[36] T. Selvan, C. Jegadheesan and P. Varthanan and K.A. Senthilkumar "A Novel FMEA approach for ranking Mould Designs in foundries". Life Science Journal. Vol. 10 $\mathrm{N}^{\mathrm{o}}$ 2, pp. 51-60. 2013.

[37] K.A. Subburaman "Modified FMEA Approach to Enhance Reliability of Lean Systems". Tesis para optar por el grado de Máster. University of Tennessee. Knoxville, Tennessee. USA. 2010. URL: http://trace. tennessee.edu/utk_gradthes/664 
[38] F. Franceschini, M. Galetto. "A new approach for evaluation of risk priorities of failure modes in FMEA". 2001. URL: http://porto. polito.it/1400121/2/1400121.pdf. Fecha de Consulta: 15 de marzo de 2016.

[39] Wang Ying-Ming, Chin Kwai-Sang, Poon, Gary Ka Kwai and Yang Jian-Bo. "Risk evaluation in failure mode and effects analysis using fuzzy weighted geometric mean". Expert Systems with Applications. Vol. 36, No 2, Part 1, p. 1195-1207. 2009. DOI: 10.1016/j.eswa.2007.11.028.

[40] M. Feitó Cespón, R. Cespón Castro y M.A. Rubio Rodríguez. "Modelos de optimización para el diseño sostenible de cadenas de suministros de reciclaje de múltiples productos". Ingeniare Revista chilena de ingeniería. Vol. $24 \mathrm{~N}^{\circ}$ 1, pp. 135-148. 2016. DOI: $10.4067 / \mathrm{S} 0718-33052016000100013$.

[41] M.P. González-Zabala y J.M. SánchezTorres. "Análisis de variables e indicadores empleados para medir la sociedad de la información". Ingeniare Revista chilena de ingeniería. Vol. $20 \mathrm{~N}^{\circ}$ 3, pp. 433-446. 2012. DOI: $10.4067 / \mathrm{S} 0718-33052012000300017$.

[42] Y. Trujillo-Casañola, A. Febles-Estrada y G. León-Rodríguez. "Modelo para valorar las organizaciones al iniciar la mejora de procesos de software". Ingeniare Revista Chilena de Ingeniería. Vol. 22 $\mathrm{N}^{\circ}$ 3, pp. 412-420. 2014. DOI: 10.4067/ S0718-33052014000300011.

[43] P. Toro, A. García, A.G. Gómez-Castro, J. Perea, R. Acero y V. Rodríguez-Estévez,
"Evaluación de la Sustentabilidad en Agroecosistemas". Archivos de Zootecnia. Vol. 59, pp. 71-94. 2010. ISSN: 1885-4494.

[44] T.J. Nahed. "Aspectos metodológicos en la evaluación de la sostenibilidad de sistemas agrosilvopastoriles". Avances en Investigación Agropecuaria. Vol. 12 No 3, pp. 3-19. 2008. ISSN: 0188789-0.

[45] International Organization for Standardization (ISO). "ISO 9004 Gestión para el éxito sostenido de una organización. Enfoque de gestión de la calidad", pp. 1-46. Fecha de actualización: 2009. Fecha de consulta: 21 de marzo de 2016. URL: www.umc.edu.ve/pdf/calidad/normasISO/ ISO_9004_ED_2009.pdf

[46] UNESCO-SCOPE. "Indicators of sustainability: Reliable tools for decision making". Fecha de actualización: 2006. Fecha de consulta: 21 de marzo de 2016. URL: http://unesdoc.unesco.org/ images/0015/001500/150005e.pdf

[47] Office for National Statistics. "Sustainable Development Indicators". Fecha de actualización: 10 de julio de 2014. Fecha de consulta: 21 de marzo de 2016. URL: www. ons.gov.uk/ons/dcp171766_368169.pdf

[48] Programa de las Naciones Unidas para el Desarrollo. "Informe sobre Desarrollo Humano". Fecha de actualización: 2015. Fecha de consulta: 24 de marzo de 2016. URL: http://hdr.undp.org/sites/default/ files/2015_human_development_report_ overview_-_es.pdf 